\title{
Computational Modeling of Psycho-physiological Arousal and Social Initiation of children with Autism in Interventions through Full-Body Interaction
}

\author{
Batuhan Sayis, Ciera Crowell, Juan Benitez, Rafael Ramirez, Narcis Pares \\ Department of Information and Communication Technologies \\ Universitat Pompeu Fabra \\ Barcelona, Spain \\ [batuhan.sayis, ciera.crowell, juanpedro.benitez, rafael.ramirez, narcis.pares]@upf.edu
}

\begin{abstract}
This study is part of a larger project that wants to foster social initiation behaviors in children with Autism Spectrum Disorder (ASD). We approach this through a fullbody interactive Mixed Reality (MR) experience that mediates a face-to-face play session between an ASD child and a non-ASD child. The goal of this study is to obtain a data model that allows us to evaluate the goodness of the MR system compared to a typical social intervention strategy based on construction tools (in this case LEGO bricks) which acts as the control condition. In this paper we present our first analysis of the arousal generated by the MR experience compared to that generated in the control condition. We address this by analyzing psychophysiological data recorded during the social interaction behaviors in the ASD child while playing with the non-ASD child. We followed a repeated-measures design with two conditions: our full-body interaction MR environment and the typical social intervention strategy based on LEGO bricks. To measure physiology, Electrocardiogram (ECG), Electrodermal Activity (EDA) and Accelerometer (ACC) data were acquired through a wearable designed by our lab. We used machine learning techniques to analyze the huge amount of multimodal data from the ASD children obtained during 18 trials ( 3 female and 15 male). As a result, we were capable of classifying social initiation behaviors of ASD children during the MR environment sessions and those occurring during the LEGO construction sessions based on the psycho-physiological data sources. This is a first sign showing that our MR system has specific properties, compared to a traditional constructionbased intervention, which potentially provide a new interesting context to intervention in ASD.
\end{abstract}

Keywords-Autism Spectrum Condition, Mixed Reality, Embodied Interaction, Social Initiation, Arousal, Psychophysiology, Computational Modeling

\section{INTRODUCTION}

Although children with Autism Spectrum Disorder (ASD) can learn to respond to social initiations started by others, they may present major difficulties in initiating these social interactions by themselves [1]. One factor that may impede the ability to initialize social interactions might be anxiety. In Mixed Reality Environments, communication and interaction can extend beyond verbal exchanges to include embodied interaction, which contributes to social perception and social understanding. Moreover, children with ASD have been found

This work has been funded by Spanish Ministry of Economy and Competitiveness under the Maria de Maeztu Units of Excellence Program (MDM-2015-0502). to have an affinity towards information and communication technologies (ICT) [2]. Practicing socialization in these environments can be a way to reduce anxiety while simultaneously training or acquiring behavioral patterns. Fullbody virtual environments strive to place the body as the center of focus, incorporating the use of gestures and nonverbal language which are key to interpersonal communication. However, we must be careful with the fact that children with ASD commonly show fluctuations in affect that may emerge from sensorial challenges and motor disturbances that can affect posture, ability to speak, and facial expressions. Therefore, the stress level of children with ASD might increase and their overt behavior can be inconsistent with their internal affective state $[3,4,5]$.

Psycho-physiology can help us better determine the children's internal state and compare it with the coding of their overt behaviors. It has become widely accepted that skin and cardiac activity, act as physiological markers that specifically reflect the emotional and cognitive states of a person $[6,7,8,9,10]$. As much as, MR, embodied interaction and psycho-physiological computing are increasingly showing potential in ASD research, the data from these systems are multimodal in nature and complex to analyze. It requires establishing a model of the complex relationship between different types of data sources. A data driven approach, such as machine learning, is ideally suited for this task. In the present study, we generated a computational model that allows us to understand the relation between the children's arousal activity and the overt social initiation behaviors shown by them; which we coded from video recordings of the play sessions.

\section{BACKGROUND}

Social interaction entails communication exchanges between individuals or groups in the form of initiations and responses [11]. Several reports have shown that high functioning children with ASD are able to sustain social interactions once initiated. However, they have lower rates of initiating social sequences and play by themselves; i.e. that they start a communication act by their own will [12].

Anxiety could be a factor that could hinder the ability to initialize social interactions. Hirstein, in 2001, showed a possible exacerbation of hyperactivity when subjects 
attempted to interact with other people [13]. Clinical observations of anxiety have closely paralleled Hirstein's behavioral and physiological observations indicating a high level of anxiety-prone symptomatology among children and adolescents with ASD [14]. Previous research in the field of ICT and Autism has highlighted the potential of technology as a basis for learning social behaviors. As unspoken social norms and expectations can be difficult to grasp by children with ASD (being non-ASD coordinated through non-verbal interaction with others [15]), practicing socialization in a virtual environment can be a way to reduce anxiety while simultaneously forming behavioral patterns. The AS Interactive Project presented by Parsons in 2006, presented an example in which individuals with ASD were trained in an assortment of virtual social sequences, such as finding a seat in a bus or a cafe [16].

There are many other Virtual Reality (VR) experiences and technologies that have been especially designed and deployed for ASD users $[17,18,19]$. Moreover, the ability to adapt a scenario in real time, according to the user's needs, highlights an additional advantage of using virtual environments. The ECHOES project [20] illustrated how a child's behavior was constantly monitored and evaluated by a system that regulated a virtual agent's disposition in response to the user model. However, the interaction designed in these projects with different interfaces and display types did not take into account the application of body attitude, proxemics, proprioceptive, or kinesthetic cues in the participants' experiences. Our belief is that this absence of embodiment does not take advantage of the full potential benefits of the virtual environments. This belief is supported by di Paolo in 2010 [21], with Embodied Cognition highlighting the significance of embodiment for social understanding and social perception. Correspondingly, the 2005 project MEDIATE, which incorporated full-body interaction, enabled child participants to engage within the environment by means of gestures, touch, and movement [22]. This research pioneered the exploration in the degree to which the affinity of ASD children with interactive settings could be beneficial in advancing and improving functioning in both communication and social domains.

Previous research has also shown that an increase in body movements in multiplayer settings increases the social nature of the gaming experience [23], thus making full-body interaction systems an interesting approach for play therapy and intervention. For example, in Pico's Adventure [24], where game play was physically controlled via a Kinect sensor, the children with ASD were asked to collaborate through coordinated movements and gestures with others. Moreover, large scale full-body interactive environments have also allowed for physical exploration and face-to-face interaction between users [25]. These kinds of technologies highlight the fact that communication and interaction extend beyond verbal exchanges to include such physical manifestations as interactional synchrony, collaborative behaviors, and proximity.

Despite these apparent advantages, in situations where embodiment is required, this communication may also be difficult for children with ASD. While there is no agreement on whether people with ASD present a different embodiment, they do show embodiment-related dysfunctions; i.e. difficulties with motor skills. Moreover, individuals with Autism can show overt, or external, behaviors that are inconsistent with their internal affective state. Even selfreports may be inaccurate for many individuals with ASD [10]; e.g. people with ASD may look relatively restful while, at the same time, show signs of physiological hyperarousal with high resting heart rate [26]. Similarly, the electrodermal activity of a person with ASD, can change between hyperarousal and hypoarousal, without any apparent changes in their overt behavior [13]. Following from this, psychophysiology could prove beneficial for research on the social activities of people with ASD. Physical symptoms like heart rate, blood pressure, and muscle tension can help detect changes in the levels of anxiety and stress of a person. Prendinger demonstrated in 2005 [27] that the measurement of EDA and Electromyogram (EMG) may be used to distinguish the change in anxiety levels of a user due to different stimulation from a virtual teacher. Another example is the project VESSI, which was a Virtual Environment System for Social Interaction designed to investigate social acceptability of virtual robots [28]. The evaluation in VESSI combined the analysis of a clinical observer and biofeedback sensor technology by identifying patterns of physiological responses across participants. Their findings were similar to the observations in social anxiety research of typical adults in real-world settings, although in this case, they specifically examined observations and physiological signals for children with ASD and TD in a virtual interaction. Liu et al. designed two computer-based cognitive tasks for children with ASD and classified anxiety at average accuracy of $79.5 \%$, using ECG, EDA, EMG, blood volume pulse (BVP), temperature, bio impedance, and heart sound [29]. A behavioral therapist tagged data instances for affective states and supervised classification was carried out using a support vector machine.

Nevertheless, physiological measures are susceptible to noise contamination and movement artifacts [30]. Indeed, special care must be taken when using both ECG and EDA since they can be severely affected by such artifacts. Furthermore, physical activity might also be correlated with the measured physiological signals [31]. Thus, physical activity becomes a critical element in wearable sensing technologies, when acquisition is conducted in ambulatory ecologically valid conditions.

\section{MATERIALS \& METHODS}

\section{A. Wearable Multichannel Psycho-physiology}

To measure physiology, ECG, EDA and ACC data were collected through a wearable designed by our lab. As a main processing unit, we used the biosignalplux multimodal platform developed by PLUX company [32]. We designed and developed this wearable specifically for children with $\mathrm{ASD}$, to collect reliable (robust to movement artifacts) physiological data in full-body interaction environments. Moreover, this wearable was designed to make the experience fun and acceptable by the children. It does not restrict movement of children as hands are free from sensors or cabling, which allows them to interact more naturally within the environment. We also considered various choices of types of sensor and electrode, and their location on the child's body since they play a very important role in the quality of psycho- 
physiological signals. We used gelled self-adhesive disposable $\mathrm{Ag} / \mathrm{AgCl}$ electrodes, as adhesive pads are another way to help reduce the impact of electrode movement on signal integrity. Previous findings on EDA measure indicate feet, fingers and shoulders as being the most responsive [33]. We therefore chose to place EDA electrodes on the shoulder in order to get a reliable signal which is least affected by movement artifacts. On the other hand, reliable ECG signal results can be obtained when both measuring electrodes are placed on locations relatively devoid of underlying muscle. Therefore, to avoid movement artifacts, electrodes may be placed on the torso. We placed two of the three lead ECG electrodes on the upper region of the chest and placed the ground electrode on the spine section of the neck. Furthermore, the position of the ACC in the wearable was aligned with the shoulder EDA electrodes so that the movement artifacts on EDA signal could be related to ACC data. The details of this wearable will be described in another paper as this design is currently in the process of patent validation.

\section{B. The MR system: Lands of Fog (Experimental Condition) \\ 1) General Description of our MR system}

The system is a Mixed Reality full-body interaction environment designed specifically for children with ASD as a space to practice social initiation with a non-ASD peer [25]. It is an installation based on a virtual environment (VE) that fosters a natural attitude of exploration and discovery of hidden virtual objects and surprises. This installation targets an experience shared by an ASD child and a non-ASD child during which they are scaffolded to socialize and collaborate.

\section{2) Installation}

The visual interface is based on a six-meter diameter floorprojected VE. The system is projected from two Full HD projectors with blended images. The children hold a physical object in their hand with which they interact with the VE. A camera tracking system tracks the objects to get their position in the playing field plane. This physical object helps children focalize their attention on the VE by acting as a guiding element, "pointer" and "placeholder" (Figure 1). This object also allows them to transform the VE, by moving or collecting virtual objects.

\section{3) Content}

The interactive experience displays an attractive range of visual and sonorous creative situations and effects to encourage the children to initiate social interaction. The VE, animations, and characters were designed with the help of children with ASD through Participatory Design Workshops [34]. The actions to be done by the children are adapted and balanced in difficulty for each child so as to generate a fair and adequate equilibrium (Figure 2).

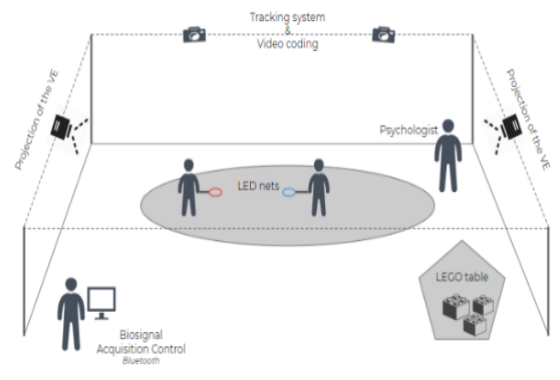

Fig. 1. Diagram of the Lands of Fog system during an experimental session
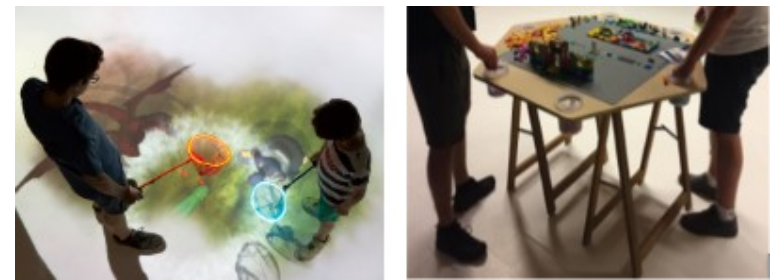

Fig. 2. Two players can collaboratively manipulate props in the scenario by bringing their nets together / A control condition included LEGO social skills training elements where children were asked to collaborate in a shared building task

\section{Non-ICT play activity: Lego Game (Control Condition)}

Following Daniel Legoff's one of the most effective and well-known play-based therapy for children with ASD, a LEGO therapy activity was modeled [35]. Legoff used LEGO brick sets to mediate communication between groups of children with ASD. Each child was given a unique role in the building process during experimental sessions so that every child was forced to co-operate and work together to achieve the final goal. The findings showed an improvement in the acquisition of social abilities, in particular in initialization of peer contact. We have adopted this strategy and created a LEGO play environment. This installation targets an experience shared by an ASD child and a non-ASD child (Figure 2).

\section{EXPERIMENTAL DESIGN \& PROCEDURE}

As a result of our collaboration with the "Multidisciplinary Unit on Autism Spectrum Disorder" of the Hospital Sant Joan de Déu, the unit provided links to the end users (i.e. highfunctioning ASD children) on a local basis in the city of Barcelona. We ran the experimental procedure with 18 ASD/non-ASD dyads. The demography was defined as children and young teenagers (8-12 years old). Participants had been formally diagnosed within ASD as determined by the Autism Diagnostic Observation Schedule (ADOS) module 3 [36], which is designed for young people with verbal fluency, with a minimum diagnosed severity of 4 . Verbal fluency being essential to achieve the level of collaboration required to play the game without the help of a psychologist or parent. As a measure to prevent problems playing or comprehending the game, both the ASD and non-ASD children, had to have a minimum IQ of 70 according to the Wechsler Intelligence Scale for Children (WISC) [37] and were screened for epilepsy.

All procedures performed were in accordance and with the 1964 Helsinki declaration and its later amendments or comparable ethical standards and ethical approval was obtained from the ethical committee of the hospital. Informed consent was obtained from the legal representatives of all participants included in the study. At least one parent had to accompany each child to the experimental trials and remain present during the entire play session. A psychologist and two researchers were also present during the trials.

Before starting the session, children were greeted first by the psychologist, who presented the researchers to the children and explained in detail, with pictograms, the activities and procedure of the session. Then, the wearable was placed on the children. We used OpenSignals software [38], developed by PLUX company, to test the connections of the different electrodes (EDA \& ECG) and reference points. Data acquisition started with the help of Biosignalplux 
Synchronization (SYNC) accessory [39] which met the multidevice synchronization need. As a preparatory step to any further behavioral instruction we used a short relaxation training to control any possible anxiety caused by the wearable and laboratory setting. Children were then instructed to enter the interactive play arena. The children with ASD played with their non-ASD partner for 15 minutes in the MR system, and with the same partner for 15 minutes in the LEGO setup. All children participated in both experimental conditions, and the order was randomized for each pair to counterbalance any learning effects. There was a 5 min break followed by a relaxation training between 2 conditions.

\section{DATA ANALYSIS}

\section{A. Preprocessing \& Feature Extraction}

\section{1) Video Coding}

Social interaction moments were mainly identified through video recording and coding for each condition of the trials. A video coding scheme was created based on Bauminger's evaluation of social-emotional understanding in children with ASD in order to code the overt behaviors of the children [39]; which was later adapted with the help of Hospital Sant Joan de Déu and the project's psychologist. The video coding scheme is founded on whether each social behavior is to be either an initiation or a response. In case the behavior was not entirely directed to someone or to a game element, it was coded as an "externalization". Externalizations such as these were prominent in the coding process, due to their potential in allowing the ASD child to initiate and create a response with their play partner. Owing to space limitation of this paper, the details of this video coding process will be briefly described here. More detail may be found in another paper that is currently under review. Essentially, the video coding was undertaken by coding each event at the instant it occurs, rather than doing an overall count for the full duration of the play session. Events separated by a minimum of five seconds were coded to make sure they are clearly differentiated. This coding system is necessary to be able to match the observed events to the real-time data obtained through the psycho-physiological sensors. However, this imposed a rough context to coders to achieve sufficiently high inter-coder agreement scores and kappa. To this end, we devised a full stepwise method represented in a flowchart for coders to strictly follow. This allowed us to achieve the desired agreement scores.

\section{2) ECG signal processing and feature extraction}

From the ECG signal recorded via the wearable, we computed the HRV which indicates the parasympathetic nervous system index [40]. This is of particular interest, given a higher parasympathetic activity through vagal tone is associated with better social functioning [40]. We computed the HRV by extracting raw ECG signal as a text file and importing it into Kubios [41]. Samples were inspected manually for artifacts. The features were processed within time windows of 30 seconds following the start of each social initiation in which there were series of RR- intervals. The RRinterval refers to the time between two R-peaks of a traditional ECG heartbeat waveform. We extracted all the features in time-domain, frequency-domain and nonlinear indices that the analysis spectrum of Kubios includes.

\section{3) EDA signal processing and feature extraction}

The EDA signal collected via the wearable consisted of a superposition of two main parts: a tonic level of skin conductance (SCL), representing the signal baseline, and superimposed phasic increases in conductance. Each of these phasic components reflects a unitary skin conductance response (SCR), reflecting the response of eccrine sweat gland activity to an external stimulus [42]. As we focused on the responses of children to the external stimuli in our activities, we evaluated the phasic components of the EDA signal. The decomposition of the EDA signal to its components was performed by means of Ledalab 3.4.9. software package for MATLAB [43]. Samples were inspected for artifacts by Automatic EDA Artifacts Identification library EDA Explorer [44] and corrected by means of interpolation. The impact of the body movements on the EDA signal did not generate strong artifacts and allowed the extraction of the features. After this phase, the deconvoluted signal was analyzed by the default peak detection algorithm. A significant peak was detected if a local maximum had a difference greater than $0.01 \mu \mathrm{S}$ from its preceding or following local minimum [45]. The phasic features were calculated within a response window (rw) up to 4 seconds in length following the social initiation onset. For EDA, the features were calculated using the default z-scale values option. We extracted all the features that the analysis spectrum of Ledalab includes.

\section{B. Computational Modeling}

As the initiation behaviors of ASD children during the experimental and control conditions might relate to the arousal activation monitored through psycho-physiological measurements, the problem to be solved was defined as a 2class classification task. The initiations taking place in the Lands of Fog (LOF) MR condition were named as "ASD LOF initiation" and the initiations occurring in the LEGO construction condition were named as "ASD LEGO initiation". The classes consisted of a balanced number of instances ( $\mathrm{N}=177$ ASD LOF initiation, $\mathrm{N}=198$ ASD LEGO initiation).

We used Weka Data Mining Software for automatic classification [46]. We applied modality fusion to integrate all incoming single modalities (EDA and HRV) into a combined single representation. In order to fuse the EDA and HRV information, we implemented feature-level fusion by using the extracted features of each modality and combined these features into a single large vector. The resulting feature set was quite large (50 features, 12 EDA features and $38 \mathrm{HRV}$ features). Therefore, it was mandatory to use a feature selection technique to find the features from both modalities that maximized the performance of the classifier. We used different feature selection strategies for our dataset. We also explored whether the model accuracies could be further enhanced by gathering more data and applying best performing algorithm in different sizes of the same dataset.

\section{1) Feature Selection}

We used three types of feature selection strategies:

a) All Features (FS1): 50 features (EDA 12 features and HRV features 38) were used as a result of feature extraction.

b) InfoGain (FS2): Automatic feature selection using filter techniques was used to acquire the most appropriate subset of features for classification. To score the features, filter techniques use a proxy measure (e.g information gain), where these features are ranked by information gain values. 
The list of the descriptors taken into account for this approach with corresponding features is presented in the InfoGain row of Table 1. The improvement in the accuracy in training the models over distinct feature subsets was also investigated, ranging from 1 feature to all features (incremental addition of features based on their information gain rank). As a result, learning curves were generated over the number of instances in order to evaluate the model's performance over incremental feature subsets (as shown in Figure 3). Figure 3 shows that the addition of each feature did not have a significant impact on the accuracy of the model. Consequently, the entire set of the features described in Table 1 was used for the InfoGain based modeling in the following sections.

c) Manual (FS3): Figure 3 shows some of the highly ranked HRV features improving the overfitting problem until the eighth iteration. When these features were investigated we saw that the feature set in the eighth iteration also includes some of the features, which best reflect the vagal tone, such as mean square of successive differences (RMSSD), pNN50 (the percentage of successive normal sinus RR intervals more than $50 \mathrm{~ms}$ ) and HF (high-frequency HRV) [47]. As a result we decided to manually create a smaller feature set keeping the two highest ranked features, MeanHR $(1 \mathrm{~min})$ (the mean heart rate) and MeanRR(ms) (the mean of Inter-beat-interval) together with RMSSD and pNN50 excluding HF which might not provide a reliable assessment of vagal tone for the rw lower than $1 \mathrm{~min}$ [40]. A similar strategy was also applied in the manual EDA feature selection step by choosing the EDA features, which represents the phasic activity most accurately [48] such as CDASCR (Average phasic driver within rw), CDAAmpSum (Sum of SCR-amplitudes of significant SCRs within rw) and CDAISCR (Area of phasic driver within rw) and creating a feature set with the highest ranking EDA feature GlobalMean (Mean SC value within rw).

\section{2) Algorithms}

We compared the results of commonly used algorithms such as Neural Networks (NN) (with one hidden layer), Support Vector Machine (SVM) (with a linear kernel), Decision Trees, K-nearest neighbor (KNN) (Number of nearest neighbors $\mathrm{K}=1$ ) and Logistic Regression using Weka Data Mining Software [46], utilizing the default parameters.

TABLE 1. The FEATURES EXTRACTED From HRV \& EDA SignALS

\begin{tabular}{|l|l|c|}
\hline Category & \multicolumn{1}{|c|}{ Features } & Signal \\
\hline \multirow{5}{*}{ All } & $\begin{array}{l}\text { Time-domain, Frequency-Domain, } \\
\text { Nonlinear Features }\end{array}$ & HRV \\
\cline { 2 - 3 } & $\begin{array}{l}\text { Continuous Decomposition (CDA) \& } \\
\text { Standard trough-to-peak (TTP) Analysis }\end{array}$ & EDA \\
\hline \multirow{5}{*}{ InfoGain } & $\begin{array}{l}\text { MeanHR(1/min), MeanRR(ms), EDR(Hz), } \\
\text { HF(ms^2), SD1(ms), pNN50(\%), } \\
\text { RMSSD(ms), NN50(count), SD2(ms), } \\
\text { STDRR(ms), Totalpower(ms^2), } \\
\text { TINN(ms), RRtriindex, LF(Hz), } \\
\text { LF(ms^2),VLF(\%),HF(\%) }\end{array}$ & HRV \\
\cline { 2 - 4 } Manual & $\begin{array}{l}\text { GlobalMean } \\
\text { MeanHR(1/min), MeanRR(ms), }\end{array}$ & EDA \\
\cline { 2 - 3 } & $\begin{array}{l}\text { GlobalMean, CDAAmpSum, CDASCR, } \\
\text { CDAISCR }\end{array}$ & HRV \\
\hline
\end{tabular}

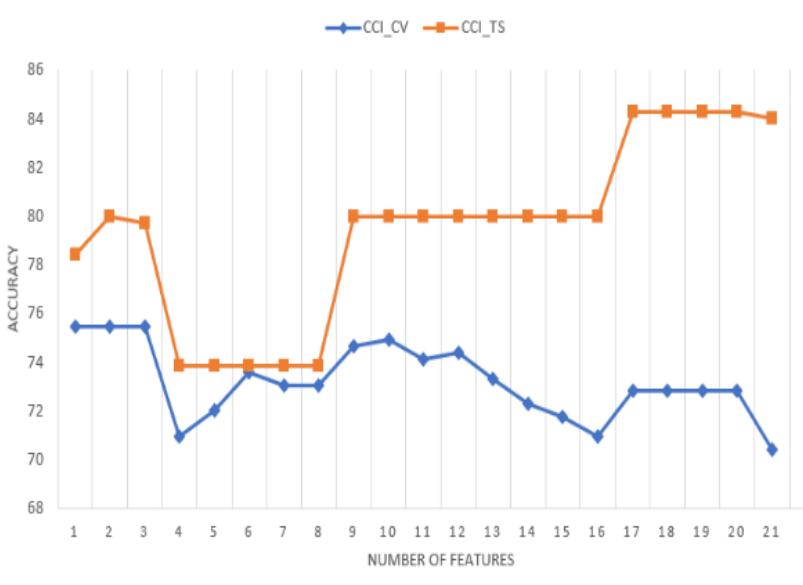

Fig. 3. Learning Curves showing the increase of the number of features organized by information gain based on comparison of Correctly Classified Instances (CCI) of Cross Validation (CV) \& Training Set (TS)

\section{RESULTS}

We generated a model for each feature selection strategy in order to investigate the effectiveness of the model. Thus, the results we present include a comparison between the feature selection strategies as well as the algorithms. We evaluated the accuracy as CCI\% using 10-fold CV (Table 2). The table below illustrates that the accuracy increased when feature selection strategies were considered (feature selection FS2 and FS3). Significant improvements were achieved by the algorithms NN and Decision Trees, 77.1 being the highest accuracy reached with the FS3 strategy with the Decision Tree Algorithm. We have also investigated whether the best performing model (Table 2) could still be improved by collecting more training data over a varying number of training instances (Figure 4).

We created different sub datasets of training instances. Each sub dataset was organized by randomly removing instances from each trial's data while preserving the uniform distribution. As shown in Figure 4, there was no significant change in the model's accuracy although there was a change in the size of the training sets.

TABLE 2. Class ClassificAtion ACCURACIES MEASURED As CCI\% FOR TRAINING SET (TS) \& 10-FOLD CROSS VALIDATION (CV)

\begin{tabular}{|l|c|c|c|c|c|c|}
\hline \multirow{2}{*}{ Classifier } & \multicolumn{2}{|c|}{ All (FS1) } & \multicolumn{2}{c|}{ InfoGain(FS2) } & \multicolumn{2}{c|}{ Manual(FS3) } \\
\cline { 2 - 7 } & TS & CV & TS & CV & TS & CV \\
\hline Baseline & 52.8 & 52.8 & 52.8 & 52.8 & 52.8 & 52.8 \\
\hline $\begin{array}{l}\text { Decision } \\
\text { Tree }\end{array}$ & 86.1 & 69.3 & 84 & 70.4 & 82.6 & $\mathbf{7 7 . 1}$ \\
\hline NN & 97.6 & 66.6 & 90.4 & $\mathbf{7 6}$ & 76.2 & 71.4 \\
\hline SVM & 73.3 & $\mathbf{7 1 . 5}$ & 70.9 & 67.7 & 70.4 & 69.3 \\
\hline $\begin{array}{l}\text { Logistic } \\
\text { Regression }\end{array}$ & 75.5 & 67.2 & 72 & 66.1 & 71.2 & 68.2 \\
\hline KNN & 85.1 & 63.7 & 84.8 & 72.2 & 80.8 & 68.8 \\
\hline
\end{tabular}




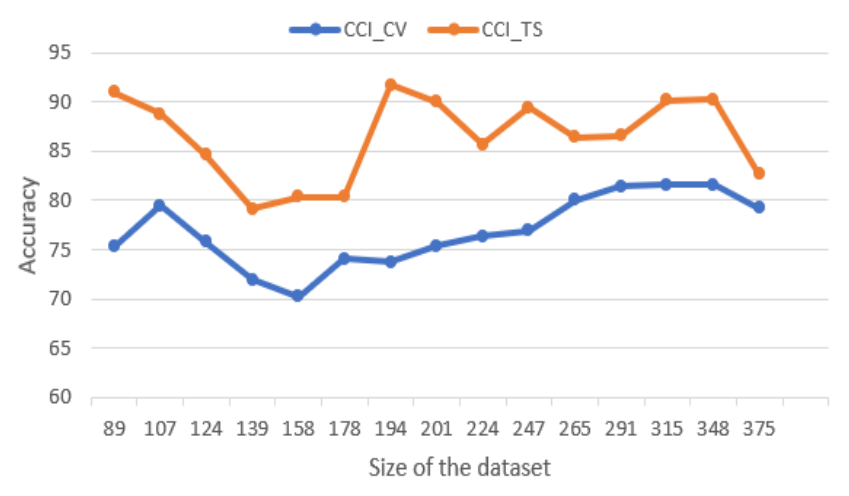

Fig. 4. Learning Curves showing the increase of training instances

\section{DISCUSSION \& CONCLUSION}

In this work, we investigated the psycho-physiological arousal activity in our full-body interaction MR experience by means of HRV and EDA recordings during social initiation behaviors in children with ASD playing with a non-ASD child. As the initiation behaviors of ASD children during the Lands of Fog MR experimental condition and LEGO control condition might relate to possible change in the arousal activity, the problem to be solved was defined as a 2-class classification task. For distinguishing between these 2 classes we used the extracted features from HRV and EDA recordings and applied different feature selection strategies which maximize the performance of the classifier. We have found that the classification accuracy increased when feature selection strategies were considered (feature selection strategies FS2 and FS3).

In FS2, the HRV feature set included some of the features which best reflect the vagal tone such as RMSSD and pNN50. On the other hand, the EDA feature set only included the feature GlobalMean while excluding the features that represent the phasic activity most accurately. A possible explanation for this contrast could be that the features selected by FS2 might have been contributing mostly to the classification of physical activity, instead of the psychophysiological arousal activity. Moreover, the GlobalMean feature was the highest-ranking feature among HRV and EDA features. This feature may reflect the thermoregulation based on physical activity [49]. Additionally, thermoregulation may play a bigger role in EDA measurement location, when comparing shoulder electrode placement to other parts of the commonly used EDA measurement locations (i.e. finger \& palm) [50]. This may be supported with our own results regarding our recordings during summer time, where we were initially unable to control the room temperature, leading us to having out of range SCL levels for two of the participants. As a result, we had to exclude these recordings in our analysis.

We are aware that physical activity might have an influence on HRV and EDA because of the reasons mentioned before. Because of this, we designed the two conditions (experimental Lands of Fog MR system and control LEGO activity) as similar as possible regarding the ambulatory nature of the activity and the exertion which the users have to go through. Therefore, this placed them in a balanced context so that they could be compared without physical activity getting in the way of our model. Hence, this feature was not found to be relevant. Moreover, when the EDA features selected included the features which are representing the phasic activity most accurately, we saw that the model's accuracy increased. The main phasic activity related EDA features formed in FS3 contributed to this classification to reach higher accuracy rates. Likewise, in FS2 (the HRV infogain feature set) we see MeanHR (1 min) feature as the highest-ranking feature. This result is likely to be related to the notion that HR positively correlates with movement. However, in the same feature set, there were a few more features automatically selected in time-domain, frequencydomain and nonlinear indices including some of the features that best reflect the vagal tone.

While creating the FS3, HRV feature set, we first took the first 2 highest ranking features into consideration although they might be related to physical activity. Additionally, we focused on adding time domain features to our FS3 feature set which might give reliable assessment of vagal tone for the response windows shorter than $1 \mathrm{~min}$. So, we added RMSSD and pNN50 time domain features into the FS3 feature set which were already highly ranked in the infogain algorithm. As a result, significant improvements were achieved by the Decision Tree algorithm, with 77.1 being the highest accuracy. We were capable of classifying the social initiation behaviors of ASD children during the Lands of Fog MR environment sessions and those occurring during the LEGO construction sessions based on psycho-physiological data sources.

On the other hand, although the number of participants involved in this study was low, the number of initiations they have done in each experience have allowed for the creation of models for classification. This result seems promising, as we were unable to improve the model accuracy significantly, despite the number of initiations increased in each sub training set. However, further work is required to establish the viability of our model including the physiological measurements which are standardized according to the quantity of the movements.

Our quest is to understand the new potential that our MR system provides as an intervention tool, compared to current typical tools, e.g. possibly achieve a lower anxiety context. In this line of research, this study is already providing a powerful model to differentiate how our users react differently in terms of psycho-physiological arousal activity in both cases. This is of vital importance to make us move towards a better understanding on how a face-to-face, full-body interactive MR system, can become a good mediator of a socialization experience between ASD and non-ASD children and become a new tool for therapists and caregivers.

\section{REFERENCES}

[1] U. Frith, Autism: A very short introduction, vol. 195. Oxford University Press, 2008.

[2] J. Brown and D. Murray, "Strategies for enhancing play skills for children with autism spectrum disorder," Educ. Train. Ment. Retard. Dev. Disabil., pp. 312-317, 2001.

[3] M. R. Leary and D. A. Hill, "Moving on: autism and movement disturbance," Ment. Retard., vol. 34, no. 1, pp. 39-53, 1996.

[4] L. Wing and A. Shah, "Catatonia in autistic spectrum disorders," Br. J. Psychiatry, vol. 176, no. 4, pp. 357-362, 2000.

[5] A. M. Donnellan, M. R. Leary, and J. P. Robledo, "Stress and the Role of Movement Differences in People with Autism," Stress Coping Autism, p. 205, 2006.

[6] P. Ekman, R. W. Levenson, and W. V Friesen, "Autonomic nervous system activity distinguishes among emotions," Science (80-. )., vol. 221, no. 4616, pp. 1208-1210, 1983.

[7] P. J. Lang, "The emotion probe: studies of motivation and attention.," Am. Psychol., vol. 50, no. 5, p. 372, 1995. 
[8] J. Healey and R. W. Picard, "Detecting stress during real-world driving tasks using physiological sensors," IEEE Trans. Intell. Transp. Syst., vol. 6, no. 2, pp. 156-166, 2005.

[9] A. Betella et al., "Inference of human affective states from psychophysiological measurements extracted under ecologically valid conditions," Front. Neurosci., vol. 8, p. 286, 2014.

[10] R. W. Picard, "Future affective technology for autism and emotion communication," Philos. Trans. R. Soc. B Biol. Sci., vol. 364, no. 1535 , pp. 3575-3584, 2009.

[11] E. Goffman, "On face-work: An analysis of ritual elements in social interaction," Psychiatry, vol. 18, no. 3, pp. 213-231, 1955

[12] M. Sigman et al., "Continuity and change in the social competence of children with autism, Down syndrome, and developmental delays," Monogr. Soc. Res. child Dev., p. i-139, 1999.

[13] W. Hirstein, P. Iversen, and V. S. Ramachandran, "Autonomic responses of autistic children to people and objects," Proc. R. Soc. London. Ser. B Biol. Sci., vol. 268, no. 1479, pp. 1883-1888, 2001.

[14] S. Kuusikko et al., "Social anxiety in high-functioning children and adolescents with autism and Asperger syndrome," J. Autism Dev. Disord., vol. 38, no. 9, pp. 1697-1709, 2008.

[15] H. De Jaegher, H. De Jaegher, M. R. Leary, and P. Practice, "Embodiment and sense-making in autism.," Front. Integr. Neurosci., vol. 7, no. March, p. 15, 2013.

[16] S. Parsons, A. Leonard, and P. Mitchell, "Virtual environments for social skills training: comments from two adolescents with autistic spectrum disorder," Comput. Educ., vol. 47, no. 2, pp. 186-206, 2006.

[17] S. Parsons and S. Cobb, "State-of-the-art of virtual reality technologies for children on the autism spectrum," Eur. J. Spec. Needs Educ., vol. 26, no. 3, pp. 355-366, 2011.

[18] N. Didehbani, T. Allen, M. Kandalaft, D. Krawczyk, and S. Chapman, "Virtual reality social cognition training for children with high functioning autism," Comput. Human Behav., vol. 62, pp. 703-711, 2016.

[19] S. N. V. Yuan and H. H. S. Ip, "Using virtual reality to train emotional and social skills in children with autism spectrum disorder," London J. Prim. Care (Abingdon)., vol. 10, no. 4, pp. 110-112, 2018.

[20] A. M. Alcorn, H. Pain, and J. Good, "Discrepancies in a virtual learning environment: something worth communicating about for young children with ASC?," in Proceedings of the 12th International Conference on Interaction Design and Children, 2013, pp. 56-65.

[21] E. Di Paolo, M. Rohde, and H. De Jaegher, "Horizons for the enactive mind: Values, social interaction, and play," in Enaction: Towards a new paradigm for cognitive science, 2010.

[22] N. Pares, P. Masri, G. Van Wolferen, and C. Creed, "Achieving dialogue with children with severe autism in an adaptive multisensory interaction: the" MEDIATE" project," IEEE Trans. Vis. Comput. Graph., vol. 11, no. 6, pp. 734-743, 2005.

[23] N. Bianchi-Berthouze, W. W. Kim, and D. Patel, "Does body movement engage you more in digital game play? and why?," in International conference on affective computing and intelligent interaction, 2007, pp. 102-113.

[24] L. Malinverni, J. Mora-Guiard, V. Padillo, L. Valero, A. Hervás, and N. Pares, "An inclusive design approach for developing video games for children with Autism Spectrum Disorder," Comput. Human Behav., vol. 71, pp. 535-549, 2017.

[25] J. Mora-Guiard, C. Crowell, N. Pares, and P. Heaton, "Lands of fog: Helping children with autism in social interaction through a full-body interactive experience," in Proceedings of the the 15th international conference on interaction design and children, 2016, pp. 262-274.

[26] M. S. Goodwin et al., "Cardiovascular arousal in individuals with autism," Focus Autism Other Dev. Disabl., vol. 21, no. 2, pp. 100-123, 2006.

[27] H. Prendinger, J. Mori, and M. Ishizuka, "Using human physiology to evaluate subtle expressivity of a virtual quizmaster in a mathematical game,” Int. J. Hum. Comput. Stud., vol. 62, no. 2, pp. 231-245, 2005.
[28] K. C. Welch, U. Lahiri, Z. Warren, and N. Sarkar, "An approach to the design of socially acceptable robots for children with autism spectrum disorders," Int. J. Soc. Robot., vol. 2, no. 4, pp. 391-403, 2010.

[29] C. Liu, K. Conn, N. Sarkar, and W. Stone, "Physiology-based affect recognition for computer-assisted intervention of children with Autism Spectrum Disorder," Int. J. Hum. Comput. Stud., vol. 66, no. 9, pp. 662-677, 2008.

[30] W. Boucsein, Electrodermal activity. Springer Science \& Business Media, 2012.

[31] R. W. Picard and J. Healey, “Affective wearables," Pers. Technol., vol. 1, no. 4, pp. 231-240, 1997.

[32] PLUX Wireless Biosignals S.A., "biosignalsplux Explorer - PLUX Store.” [Online]. Available: https://store.plux.info/kits/215-biosignalsexplorer-820201001.html. [Accessed: 17-Apr-2019].

[33] M. van Dooren and J. H. Janssen, "Emotional sweating across the body: Comparing 16 different skin conductance measurement locations," Physiol. Behav., vol. 106, no. 2, pp. 298-304, 2012.

[34] L. Malinverni, J. Mora-Guiard, and N. Pares, "Towards methods for evaluating and communicating participatory design: A multimodal approach,” Int. J. Hum. Comput. Stud., vol. 94, pp. 53-63, 2016.

[35] D. B. LeGoff, "Use of LEGOC as a therapeutic medium for improving social competence," J. Autism Dev. Disord., vol. 34, no. 5, pp. 557571, 2004.

[36] C. Lord et al., "Austism diagnostic observation schedule: A standardized observation of communicative and social behavior," J. Autism Dev. Disord., vol. 19, no. 2, pp. 185-212, 1989.

[37] D. Wechsler, "Wechsler intelligence scale for children.," 1949.

[38] Osr, "OpenSignals (r)evolution Software Data Sheet," 2016.

[39] N. Bauminger, "The facilitation of social-emotional understanding and social interaction in high-functioning children with autism: Intervention outcomes," J. Autism Dev. Disord., vol. 32, no. 4, pp. 283-298, 2002.

[40] M. Malik et al., "Heart rate variability: Standards of measurement, physiological interpretation, and clinical use," Eur. Heart J., vol. 17, no. 3, pp. 354-381, 1996.

[41] M. P. Tarvainen, J.-P. Niskanen, J. A. Lipponen, P. O. Ranta-Aho, and P. A. Karjalainen, "Kubios HRV-heart rate variability analysis software," Comput. Methods Programs Biomed., vol. 113, no. 1, pp. 210-220, 2014.

[42] S. for P. R. A. H. C. on E. Measures et al., "Publication recommendations for electrodermal measurements," Psychophysiology, vol. 49, no. 8, pp. 1017-1034, 2012.

[43] M. Benedek and C. Kaernbach, "Decomposition of skin conductance data by means of nonnegative deconvolution," Psychophysiology, vol. 47, no. 4, pp. 647-658, 2010.

[44] N. Taylor, S., and Jaques, "EDA Explorer." [Online]. Available: https://eda-explorer.media.mit.edu/. [Accessed: 18-Apr-2019].

[45] J. J. Braithwaite, D. G. Watson, R. Jones, and M. Rowe, “A guide for analysing electrodermal activity (EDA) \& skin conductance responses (SCRs) for psychological experiments," Psychophysiology, vol. 49, no. 1, pp. 1017-1034, 2013.

[46] M. Hall, E. Frank, G. Holmes, B. Pfahringer, P. Reutemann, and I. H. Witten, "The WEKA data mining software: an update," ACM SIGKDD Explor. Newsl., vol. 11, no. 1, pp. 10-18, 2009.

[47] H. Otzenberger et al., "Dynamic heart rate variability: a tool for exploring sympathovagal balance continuously during sleep in men," Am. J. Physiol. Circ. Physiol., vol. 275, no. 3, pp. H946-H950, 1998.

[48] Ledalab Documentation. (n.d.). Retrieved from http://www.ledalab.de/documentation.htm

[49] J. Cacioppo, L. G. Tassinary, and G. G. Berntson, The Handbook of Psychophysiology, vol. 44. 2007.

[50] G. Turpin, P. Shine, and M. H. Lader, "Ambulatory electrodermal monitoring: effects of ambient temperature, general activity, electrolyte media, and length of recording.," Psychophysiology, 1983. 